\title{
P01-1-112 Poster session
}

\section{The activation of Nrf2 contributes to the inhibitory effect of salvianolic acid A on NF-kappaB signaling in BV2 microglia cells}

Junke Song ${ }^{1,2}$, Wen Zhang ${ }^{1,2}$, Guanhua Du ${ }^{1,2}$

${ }^{1}$ Institute of Materia Medica, Chinese Academy of Medical Sciences and Peking Union Medical College, China, ${ }^{2}$ Beijing Key Laboratory of Drug Target Identification and Drug Screening, China

Aim: Neurodegenerative diseases are closely related to neuroinflammation. Nrf2, as an antioxidant factor, has been shown to possess anti-inflammation effects via NF-kappaB inhibition. Salvianolic acid A (SalA) was isolated from Salvia miltiorrhiza Bunge, a plant with the reported effects of anti-inflammation. However, the anti-inflammation effects and mechanisms of SalA on LPS-stimulated BV2 microglia cells remain unknown. The aim of this study was to clarify whether Nrf2 activation contributes to SalA mediated NF-kappaB inhibition in BV2 microglia cells.

Methods: MTT assay was carried out to detect cell viability. The levels of TNF-alpha and IL-1beta; were detected by ELISA method. The expressions of p-NF-kappaB p65, p-IkappaB, Nrf2 and HO-1 proteins were studied by western blot analysis. The activation of NF-kappaB and nuclear translocations of Nrf2 were studied by immunofluorescence assay. The specific Nrf2 siRNA was used to clarify the interaction between Nrf2 and NF-kappaB p65.

Results: LPS induced inflammatory cytokines expression and the phosphorylation and nuclear translocation of NF-kappaB p65. SalA reversed the inflammatory response induced by LPS and inhibited NF-kappaB activation. Furthermore, SalA upregulated the expression of Nrf2, HO-1 and NQO1. But the anti-inflammation and NF-kappaB inhibition effects of SalA were attenuated by the transfection of specific Nrf2 siRNA.

Conclusion: SalA inhibited LPS-induced inflammatory response, and downregulated NF-kappaB phosphorylation and nuclear translocation, which was mediated by the activation of Nrf2 signaling pathway in BV2 microglia cells.

Acknowledgment: This work was supported by grants from The CAMS Innovation Fund for Medical Sciences (2017-I2M -1-010); National Natural Science Foundation of China (No. 81603100); The open project of State Key Laboratory of Toxicology and Medical Countermeasures (TMC201510). 